\title{
Cytomegalovirus Enterocolitis in a Term Neonate
}

\author{
Siva Prasad Vyasam ${ }^{1}$, Thangaraj Abiramalatha ${ }^{1}$, Nalapalu Srinivasan Hema ${ }^{2}$ and Niranjan Thomas ${ }^{1}$ \\ From Departments of ${ }^{1}$ Neonatology and ${ }^{2}$ Pediatrics, Christian Medical College, Vellore, Tamil Nadu, India.
}

Correspondence to: Prof. Niranjan Thomas, Department of Neonatology, $3^{\text {rd }}$ floor, ISSCC Building, CMC, Vellore, Tamil Nadu, India. niranjan@cmcvellore.ac.in Received: August 21, 2016; Initial review: May 17, 2017; Accepted: December 01, 2017.

\begin{abstract}
Background: Cytomegalovirus (CMV) enterocolitis is rare in term neonates. Case characteristics: A term newborn with persistent pneumonia from birth developed enterocolitis on day 18 of life. Outcome: Polymerase chain reaction (PCR) for CMV DNA was positive in urine sample. Antiviral therapy for six weeks resulted in successful treatment without any stricture formation. Message: CMV enterocolitis should be considered as a differential diagnosis in atypical cases of necrotizing enterocolitis in neonates.
\end{abstract}

Keywords: Diagnosis, Polymerase chain reaction, Necrotizing enterocolitis, Treatment.

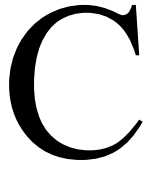
ytomegalovirus (CMV) is the commonest congenital infection with a prevalence varying from $0.2-2.2 \%$ in different parts of world [1]. Symptomatic congenital CMV infection may present in $10 \%$ of affected newborns with fetal growth restriction, hepatosplenomegaly, microcephaly, seizures, ocular defects, thrombocytopenia and conjugated hyperbilirubinemia [1]; gastrointestinal symptoms are uncommon. We report a term baby with CMV enterocolitis, who was successfully treated with antiviral therapy.

\section{Case Report}

A male neonate born at 37 weeks gestation with birth weight of 2600 gram presented at 4 hours of life with respiratory distress since birth. There was no history of meconium-stained liquor or need for resuscitation at birth. At admission, he had severe respiratory distress with a Downe score of 9 . His chest radiograph was suggestive of bilateral pneumonia.

The baby was started on continuous positive airway pressure (CPAP) of $6 \mathrm{~cm}$ with $60 \% \mathrm{FiO}_{2}$, intravenous (IV) fluids and antibiotics. In view of impending respiratory failure, he was intubated and started on mechanical ventilation. He required ventilatory support for five days followed by CPAP for six days after which he was weaned to high-flow oxygen through hood. Blood culture was sterile and he completed two weeks of IV antibiotics. His supplemental oxygen requirement persisted. Echocardiography was normal. He was started on gavage feeds with expressed breast milk from day five, which was graded up to full feeds by day twelve of life.

On day 18 of life, the baby developed fever, tachycardia, abdominal distension and right iliac fossa tenderness. A plain abdominal radiograph showed dilated bowel loops. Ultrasound abdomen revealed moderate ascites. Blood counts, serum electrolytes and blood gas were normal. A provisional diagnosis of necrotising enterocolitis (NEC) was made. Enteral feeds were discontinued and IV antibiotics were restarted. Baby continued to have abdominal distension with bilious aspirates, with no improvement after 72 hours of antibiotics. Blood culture was sterile and sepsis screen was negative. Stool ELISA for rotavirus antigen was also negative.

A possibility of CMV infection was kept in view of persistent pneumonia with enterocolitis, with normal metabolic and sepsis screen and poor response to antibiotics. Urine CMV PCR was positive. Liver and kidney function tests were normal. Cranial ultrasound showed left periventricular calcification. Ophthalmic evaluation was normal. Hearing screening by automated auditory brainstem response (AABR) done at 6 weeks of age was normal. Mother's CMV serology could not be done.

The baby was started on antiviral therapy on day 21 of life with IV Gancyclovir $6 \mathrm{mg} / \mathrm{kg} /$ dose twice a day, subsequent to which clinical improvement was noted. Oxygen was discontinued on day 24 of life. He was restarted on enteral feeds on day 25 , which he tolerated well. Gancyclovir was continued for 2 weeks, followed by oral Valgancyclovir in a dose of $16 \mathrm{mg} / \mathrm{kg} /$ dose twice a day for 4 weeks. He was discharged on day 36 of life. At 1 year follow-up, his growth and development were normal. His neurological examination, hearing and vision were normal. 


\section{DisCUSSION}

Neonatal CMV infection can be congenital (transplacental transmission), perinatal (through cervical secretions) or postnatal (through breast milk or blood products) [1]. Since the incubation period is 4-12 weeks, almost all cases of perinatal or postnatal CMV infection present after the newborn period. In our case, the clinical presentation and positive urine CMV PCR within the first 3 weeks of life with periventricular calcification suggested in utero transmission and congenital infection.

CMV enterocolitis is a common entity in immunocompromised hosts. It may present as intractable diarrhea in healthy infants at 4-12 weeks of age [2]. During the neonatal period, perinatal/postnatal CMV infection presents as NEC in premature infants $[3,4]$. CMV enterocolitis in a term neonate is rare. Pathologically, CMV causes ulcers in the intestine, which result in strictures on healing [5]. This is in contrast to the classical NEC, which causes transmural gangrene of the bowel wall and perforation. Reports of infants presenting later with intestinal strictures suggest that CMV enterocolitis is often a missed diagnosis in the newborn period $[5,6]$.

CMV DNA-PCR of urine, blood or saliva is the most commonly used diagnostic test. Stool PCR can be used as a non-invasive test in enterocolitis [7]. Detection of intramuscular cytomegalic inclusion bodies in intestinal biopsy is diagnostic [6]. In our case, biopsy was not done as the baby responded promptly to antiviral therapy and did not require surgery.

There is no consensus to guide the treatment of congenital CMV infection. The trials conducted by Collaborative Antiviral Study Group (CASG) recommend six weeks therapy with IV Gancyclovir or six months therapy with enteral Valgancyclovir in infants with congenital CMV infection and central nervous system manifestations (CNS) or end organ dysfunction, without separate mention of CMV enterocolitis [7,8]. Successful treatment of CMV enterocolitis with IV or enteral Gancyclovir and enteral Valgancyclovir has been previously documented $[9,10]$. Similar response was seen in our case. The prognosis of symptomatic congenital CMV infection is poor, with long-term sequale reported in $60-80 \%$ cases in the form of hearing loss, intellectual disability and visual disturbances [1]. Our baby did not have any neurosensory impairment till one year of age. The additive effect of the antiviral therapy given to the baby in preventing long-term sequalae cannot be entirely dismissed. Nigro, et al. [10] reported normal growth and neurodevelopment till 6 years of age in three children who had CMV enterocolitis in early infancy and had been treated with oral Gancyclovir therapy.
There is a recent debate as to whether CMV is the causative agent or a misleading trail in neonates presenting with enterocolitis [6]. In our case, the dramatic clinical response to Gancyclovir suggests that CMV was probably the causative agent rather than an incidental diagnosis. We suggest that CMV infection should be considered as a differential diagnosis in all the atypical cases of NEC in neonates. Early diagnosis and antiviral therapy with Gancyclovir/Valgancyclovir may avoid long-term sequelae.

Contributors: SPV and TA: Drafting the manuscript, literature review and patient management; NSH: Literature search, editing the manuscript and managing the patient; NT: Revising the article critically for important intellectual content. All authors approved the final version of manuscript.

Funding: None; Competing interest: None stated.

\section{REFERENCES}

1. Britt W. Cytomegalovirus. In: Wilson $\mathrm{CB}$, Nizet $\mathrm{V}$, Maldonado YA, Remington JS, Kl ein JO, eds. Remington and Klein's Infectious Diseases of the Fetus and Newborn Infant. China: Elsevier Saunders; 2016. p. 724-81.

2. Refai Z, Nicholls S, Garg A. Infant death due to CMV enterocolitis. BMJ Case Rep. 2012;2012.

3. Gessler P, Bischoff GA, Wiegand D, Essers B, Bosart W. Cytomegalovirus-associated necrotizing enterocolitis in a preterm twin after breastfeeding. J Perinatol. 2004;24: 124-6.

4. Tran L, Ferris M, Norori J, Stark M, Craver R, Dowd S, et al. Necrotizing enterocolitis and cytomegalovirus infection in a premature infant. Pediatrics. 2013;131:e318-22.

5. Yeung F, Chung PHY, Wong KKY, Tam PWH. Cytomegalovirus-associated colitis mimicking necrotizing enterocolitis-A near miss diagnosis of neonatal colonic stricture. J Pediatr Surg Case Rep. 2014;2:459-61.

6. Unlusoy Aksu A, Sarý S, Karabulut R, Ekinci O, Daigic B. Jejunal stricture in a premature infant: Is cytomegalovirus the causative pathogen or a superinfection? Turk J Gastroenterol. 2013;24:273-6.

7. Kimberlin DW, Lin C-Y, Sanchez PJ, Demmler GJ, Dankner W, Shelto M, et al. Effect of ganciclovir therapy on hearing in symptomatic congenital cytomegalovirus disease involving the central nervous system: a randomized, controlled trial. J Pediatr. 2003;143:16-25.

8. Kimberlin DW, Jester PM, Sánchez PJ, Ahmed A, AravBoger R, Michaels M et al. Valganciclovir for symptomatic congenital cytomegalovirus disease. N Engl J Med. 2015;372:933-43.

9. Irizarry K, Honigbaum S, Demmler-Harrison G, Rippel S, Wilsey M. Successful treatment with oral valganciclovir of primary CMV enterocolitis in a congenitally infected infant. Fetal Pediatr Pathol. 2011;30:437-41.

10. Nigro G, Pietrobattista A, Divito S, Gambarara M. Oral gancyclovir therapy for immunocompetent infants with cytomegalovirus-associated hemorrhagic or intractable enterocolitis. J Pediatr Gastroenterol Nutr. 2010;50:111-3. 